\title{
Inonotus obliquus extract alleviates myocardial ischemia/reperfusion injury by suppressing endoplasmic reticulum stress
}

\author{
YI WU $^{1}$, HEMING CUI ${ }^{1}$, YUYING ZHANG ${ }^{1}$, PING YU ${ }^{1}$,

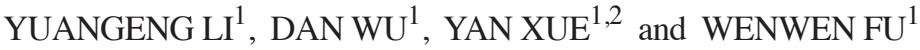 \\ ${ }^{1}$ Department of Pharmacology, School of Pharmaceutical Sciences, Jilin University; \\ ${ }^{2}$ Department of Burn Surgery, The First Hospital of Jilin University, Changchun, Jilin 130021, P.R. China
}

Received July 17, 2020; Accepted November 10, 2020

DOI: $10.3892 / \mathrm{mmr} .2020 .11716$

\begin{abstract}
Inonotus obliquus (IO) is an edible fungus that exerts various biological functions, including anti-inflammatory, antitumor and immunomodulatory effects. The present study was designed to investigate the role of IO extract (IOE) in myocardial ischemia/reperfusion (MI/R) and determine the exact molecular mechanisms. The left anterior descending coronary artery was ligated to establish the MI/R injury model in rats. IOE exhibited a novel cardioprotective effect, as shown by improvement in cardiac function and decrease in infarct size. Pretreatment with IOE activated antioxidant enzymes in cardiomyocytes, including glutathione peroxidase, superoxide dismutase and catalase. IOE pretreatment also induced the upregulation of NAD-dependent protein deacetylase sirtuin-1 (SIRT1) and downregulation of glucose-regulated protein 78, phosphorylated (p-) protein kinase R-like endoplasmic reticulum kinase, $\mathrm{p}$-eukaryotic translation initiation factor 2 subunit $\alpha, \mathrm{C} / \mathrm{EBP}$ homologous protein and caspase-12. Furthermore, IOE alleviated endoplasmic reticulum (ER) stress-induced apoptosis in cardiomyocytes by decreasing the mRNA levels of caspase-12. IOE inhibited apoptosis induced by overexpression of pro-caspase-9 and pro-caspase-3. In summary, IOE pretreatment protects the heart against MI/R injury through attenuating oxidative damage and suppressing ER stress-induced apoptosis, which may be primarily due to SIRT1 activation.
\end{abstract}

Correspondence to: Dr Wenwen Fu, Department of Pharmacology, School of Pharmaceutical Sciences, Jilin University, 1266 Fujin Road, Changchun, Jilin 130021, P.R. China

E-mail: fww@jlu.edu.cn

Dr Yan Xue, Department of Burn Surgery, The First Hospital of Jilin University, 71 Xinmin Street, Changchun, Jilin 130021, P.R. China E-mail: xueyan0905@jlu.edu.cn

Key words: Inonotus obliquus extract, myocardial ischemia/ reperfusion injury, NAD-dependent protein deacetylase sirtuin-1, endoplasmic reticulum stress, apoptosis

\section{Introduction}

Cardiovascular disease is a challenging disease with high morbidity and mortality worldwide (1). Myocardial ischemia/reperfusion (MI/R) injury is the primary risk factor in the pathological process of heart disease. Although reperfusion is the most efficient way to mimic tissue damage, the re-establishment of the blood supply is known to induce various adverse events (2). In addition, $\mathrm{MI} / \mathrm{R}$ also induces endoplasmic reticulum (ER) stress and activates apoptosis-related signaling pathways (3).

The ER serves a number of roles in protein synthesis, lipid biosynthesis and detoxification. When normal ER function is disrupted by various cellular disturbances, unfolded and misfolded proteins accumulate, resulting in a condition known as ER stress (4). The alteration of redox homeostasis results in excessive generation of reactive oxygen species (ROS), which subsequently causes ER stress (5). ER stress promotes the dissociation of the ER chaperone glucose-regulated protein 78 (GRP78) from transmembrane proteins and triggers their activation (6). Protein kinase R-like endoplasmic reticulum kinase (PERK) is one of transmembrane proteins and dimerizes under ER stress, which phosphorylates eukaryotic translation initiation factor 2 subunit $\alpha(\operatorname{eIF} 2 \alpha)$ (7). eIF2 $\alpha$ mediates the transcription of activating transcription factor-4 (ATF4), which interacts with the pro-apoptotic factor C/EBP homologous protein (CHOP) (8). Additionally, ER stress induces cell apoptosis by activating the caspase family cascade (9).

NAD-dependent protein deacetylase sirtuin-1 (SIRT1), known as one of the mammalian homologues of yeast Sir2, is a histone deacetylase and regulates various biological processes, such as the inflammatory reaction, oxidative stress and apoptosis $(10,11)$. A previous study demonstrated that SIRT1 overexpression protects cardiomyocytes against MI/R injury from ER stress (12). The protective effect is due to the activation of the PERK/eIF2 $\alpha$ pathway, which is associated with cardiomyocyte apoptosis. Hence, SIRT1 may be a potential target to treat cardiac pathologies by attenuating ER stress.

Inonotus obliquus ( $\mathrm{IO}$ ) is a fungus belonging to the Hymenochaetaceae family of Basidiomycetes, which grows 
on birch trees (13). The main bioactive compounds of IO are polysaccharides, polyphenols, melanin and triterpenes (14). IO exerts various biological functions, including anti-inflammatory, antitumor and immunomodulatory effects $(15,16)$. However, its effects on cardiovascular diseases has been rarely studied. The current study was designed to investigate the role of IO extract (IOE) in MI/R injury and determine the exact molecular mechanisms.

\section{Materials and methods}

Ethanol extraction of IO. IO was collected from Jilin Province, Northeastern China and identified by Professor GuangShu Wang (Jilin University). A total of 2,500 g IO powder was weighed, soaked in 12.51 distilled water overnight at room temperature and extracted at $80^{\circ} \mathrm{C}$ for $2.5 \mathrm{~h}$. The supernatant was collected after filtration using three layers of gauze, and the extraction was repeated twice in the same manner. The filtrate was concentrated by rotary evaporation, dried at $60^{\circ} \mathrm{C}$, crushed and passed through an 80-mesh sieve to obtain the preliminary extract. Next, $12.5170 \%$ ethanol was added to the preliminary extract obtained from water extraction (extracted at $75^{\circ} \mathrm{C}$ for $2.5 \mathrm{~h}$ ). The supernatant was gathered by filtering through three layers of gauze, and the extraction was repeated twice in the same manner. The filtrates were collected and concentrated by rotary evaporation, then dried at $60^{\circ} \mathrm{C}$, crushed and passed through an 80-mesh sieve, and $127.9 \mathrm{~g}$ IOE was obtained. The extraction rate of IOE was $5.12 \%$ according to the following formula: Extraction rate $=$ weight of $\mathrm{IOE} /$ weight of $\mathrm{IO}$ powder. Meanwhile, the main composition of IOE was assessed using a T-6 Series UV-Vis spectrophotometer (Beijing Purkinje General Instrument Co., Ltd.). The contents of total flavonoid, total saponin, polysaccharide and polyphenol were calculated using respective standard curves $(\mathrm{y}=0.0117 \mathrm{x}+0.0554$, $\mathrm{R}^{2}=0.9998 ; \mathrm{y}=0.0301 \mathrm{x}+0.0871, \mathrm{R}^{2}=0.9992 ; \mathrm{y}=16.253 \mathrm{x}+0.1019$, $\mathrm{R}^{2}=0.9999$ and $\left.\mathrm{y}=97.266 \mathrm{x}+0.1984, \mathrm{R}^{2}=0.9996\right)$ with the percentage of $1.76,1.06,0.57$ and $1.07 \%$.

Animals. Wistar rats (female, 250-280 g) were purchased from Changchun Yisi Experimental Animal Co., Ltd., and raised in a controlled environment $\left(22-24^{\circ} \mathrm{C} ; 55-60 \%\right.$ humidity and $12 / 12-\mathrm{h}$ light/dark cycle) with free access to food and water. Animals were treated according to the Guide for the Care and Use of Laboratory Animals (US National Institutes of Health) (17) and the Committee for the Care and Use of Laboratory Animals of Jilin University (Changchun, China). The animal experiments of the present study were approved by the Ethical Committee for Experimental Animals, School of Pharmaceutical Sciences, Jilin University (approval no. 20190049).

Experimental design. A total of 150 female rats were randomly assigned into five groups ( $\mathrm{n}=30$ ): i) Non-MI/R sham group (Sham); ii) MI/R group (MI/R); iii) MI/R+IOE $150 \mathrm{mg} / \mathrm{kg}$ group (MI/R-IOE150); iv) MI/R+IOE 300 mg/kg group (MI/R-IOE300) and v) MI/R+IOE $600 \mathrm{mg} / \mathrm{kg}$ group (MI/R-IOE600). The animals in the treatment groups were intragastrically administered with IOE $(150,300$ or $600 \mathrm{mg} / \mathrm{kg}$ ) for 7 consecutive days. The rats of the Sham and MI/R groups were intragastrically administered with $0.5 \%$ carboxymethylcellulose sodium for 7 consecutive days. Surgical procedures were performed on rats in the Sham group, but without left anterior descending (LAD) coronary artery ligation. In other groups, LAD ligation was performed on the hearts for $2 \mathrm{~h}$ and then reperfused for $2 \mathrm{~h}$.

Experimental model establishment. At day 7, 3\% sodium pentobarbital $(30 \mathrm{mg} / \mathrm{kg}$ body weight, intraperitoneal injection) was used to anesthetize the rats after $1 \mathrm{~h}$ of administration. Rat hearts were exteriorized via open-chest surgery. A 6/0 nylon suture was put to make a slip knot around the LAD coronary artery and induce myocardial ischemia. After $2 \mathrm{~h}$ of ligation, the ligature was loosened to restore myocardial blood supply for myocardial reperfusion. Rats in the Sham group were subjected to the same procedure, but without ligation. Reperfusion was performed for $2 \mathrm{~h}$. The rats were anesthetized by intraperitoneal injection with $3 \%$ sodium pentobarbital ( $30 \mathrm{mg} / \mathrm{kg}$ body weight). Then, $\sim 8 \mathrm{ml}$ volume of blood was taken from the abdominal aorta of the unconscious rats for painless euthanasia. Death was confirmed when respiratory and cardiac arrest was observed, following which, cardiac tissues were harvested for further experiments.

Cardiac function assessment. After $2 \mathrm{~h}$ of reperfusion, $3 \%$ sodium pentobarbital ( $30 \mathrm{mg} / \mathrm{kg}$ body weight, intraperitoneal injection) was used to anesthetize the rats. Once anesthetized, the thoracic area was shaved and covered with ultrasonic transmission gel. A portable echocardiography machine (GE Vivid I; GE Healthcare) was used to evaluate the cardiac function of rats. The left ventricular (LV) ejection fraction (EF), LV fractional shortening (FS), LV internal dimensions at diastole (LVIDd) and LV internal diameter systole (LVIDs) were evaluated. An observer who was blinded to the experimental design measured these parameters in three consecutive cardiac cycles.

Determination of myocardial infarction size (MIS). After $2 \mathrm{~h}$ of reperfusion, the heart tissues were excised to perform MIS measurements. Five pieces of the left ventricle parallel to the atrioventricular groove were transected, immersed in $0.5 \%$ nitro blue tetrazolium chloride (NBT) phosphate buffer and stained for $1 \mathrm{~min}$ in a $37^{\circ} \mathrm{C}$ water bath. Normal myocardial tissues were stained dark red, while ischemic myocardial tissues were pale. The ischemic myocardial tissue was excised and weighed. The MIS was calculated as follows: Ischemic myocardial weight/wet weight of the left ventricle x100\%.

Determination of cardiac enzymes. A total of $2 \mathrm{ml}$ blood was collected after $2 \mathrm{~h}$ of reperfusion. Serum was separated from the blood samples by centrifugation $\left(2,000 \mathrm{x} \mathrm{g}, 10 \mathrm{~min}, 4^{\circ} \mathrm{C}\right)$ and used for the evaluation of creatine kinase-MB (CK-MB; cat. no. H197; Nanjing Jiancheng Bioengineering Institute), aminotransferase (AST; cat. no. C010-1-1; Nanjing Jiancheng Bioengineering Institute) and lactate dehydrogenase (LDH; cat. no. A020-2-2; Nanjing Jiancheng Bioengineering Institute) activities using respective diagnostic kits in accordance with the manufacturer's instructions.

Determination of oxidative stress levels in myocardial tissue. After $2 \mathrm{~h}$ of reperfusion, rat hearts were excised to prepare heart homogenates. Prepared myocardial tissues were excised and homogenized (10\%) in ice-cold physiological saline solution using a Tissue-Tearor (Bio Spec Products, Inc.). 
The supernatant was separated from heart homogenates by centrifugation $\left(3,500 \mathrm{x} \mathrm{g}, 15 \mathrm{~min}, 4^{\circ} \mathrm{C}\right)$ and used for the evaluation of superoxide dismutase (SOD; cat. no. A001-1-2; Nanjing Jiancheng Bioengineering Institute), malondialdehyde (MDA; cat. no. A003-1-1; Nanjing Jiancheng Bioengineering Institute), glutathione peroxidase (GSH-px; cat. no. A005-1-2; Nanjing Jiancheng Bioengineering Institute) and catalase (CAT; cat. no. A007-2-1; Nanjing Jiancheng Bioengineering Institute) levels using respective diagnostic kits in accordance with the manufacturer's instructions.

Histological examination. After $2 \mathrm{~h}$ of reperfusion, rat hearts were quickly harvested. and the blood was expelled. Hearts were fixed in $4 \%$ paraformaldehyde solution for $24 \mathrm{~h}$ at room temperature. After rinsing with water, heart tissues were dehydrated with gradient ethanol solution and embedded in paraffin. Subsequently, heart tissues were cut into 3-5 $\mu \mathrm{m}$-thick slices and stained using a hematoxylin and eosin (H\&E) kit for $3 \mathrm{~min}$ at room temperature. An optical microscope was used to observe morphological changes. The degree of cardiac damage was assessed by an independent researcher blind to the experimental design.

TUNEL assay. Myocardial apoptosis was evaluated using a TUNEL assay kit (cat. no. 11684817910; Sigma-Aldrich; Merck KGaA). Heart tissue was fixed in $4 \%$ paraformaldehyde solution for $24 \mathrm{~h}$ at room temperature, embedded in paraffin, then sectioned on slides at 3-5 $\mu$ m-thickness. TUNEL (1:9 mixed in equilibration buffer) staining of apoptotic cells (green fluorescence). DAPI $(5 \mu \mathrm{g} / \mathrm{ml})$ was used to dye the nuclei for $5 \mathrm{~min}$ (blue fluorescence). The slides were then sealed with $50 \%$ glycerin. Five randomly selected fields of view were observed and apoptotic cells of each section were counted under an ECLIPSE 80i fluorescence microscope (Nikon Corporation). The cardiomyocyte apoptotic index (AI) was counted as follows: Number of TUNEL-positive nuclei/the number of total nuclei $\mathrm{x} 100 \%$.

Reverse transcription-quantitative $(R T-q) P C R$. Total RNA was extracted from cardiac tissues using TRIzol ${ }^{\circledR}$ reagent (Invitrogen; Thermo Fisher Scientific, Inc.), quantified with the T-6 Series UV-VIS spectrophotometer (Beijing Purkinje General Instrument Co., Ltd.) and reverse transcribed into cDNA using a Hifair ${ }^{\circledR}$ II First Strand cDNA Synthesis Kit (cat. no. 11120ES60; Shanghai Yeasen Biotechnology Co., Ltd.). RT was carried out at $42^{\circ} \mathrm{C}$ for $15 \mathrm{~min}$, then $85^{\circ} \mathrm{C}$ for $15 \mathrm{sec}$.Subsequently,qPCR was performed with sequence-specific primers using the SYBR Green MasterMix kit (cat.no.11202ES03; Shanghai Yeasen Biotechnology Co., Ltd.). The thermocycling conditions consisted of $40-50$ cycles at $95^{\circ} \mathrm{C}$ for $10 \mathrm{sec}, 55^{\circ} \mathrm{C}$ for $20 \mathrm{sec}$ and $72^{\circ} \mathrm{C}, 20 \mathrm{sec}$. The following primer pairs were used for qPCR: Caspase-12 forward, 5'-CAGCACATTCCTG GTGTTTAT-3' and reverse, 5'-GACTCTGGCAGTTACGGTT GTT-3' and $\beta$-actin forward, 5'-GCACCGCAAATGCTTCT AGG-3' and reverse, 5'-AAAGGGTGTAAAACGCAGCTC-3'. Data were calculated using the $2^{-\Delta \Delta \mathrm{Cq}}$ method (18) with the mRNA levels of $\beta$-actin as an internal control.

Western blot assay. Myocardial tissues were homogenized in lysis buffer (cat. no. P0013; Beyotime Institute of Biotechnology) and protease inhibitor cocktail on ice and purified by centrifugation at $14,000 \times \mathrm{g}$ for $15 \mathrm{~min}$ at $4^{\circ} \mathrm{C}$. The protein concentration was determined using the BCA method. A total of $40 \mu \mathrm{g}$ protein was loaded onto $10-12 \%$ gels and resolved via SDS-PAGE. Separated proteins were then transferred to a PVDF membrane for $2 \mathrm{~h}$. PVDF membranes were blocked for $1.5 \mathrm{~h}$ in $5 \%$ bovine serum albumin (cat. no. P0252; Beyotime Institute of Biotechnology) at room temperature and washed three times with TBS+0.2\% Tween-20. Membranes were incubated overnight at $4^{\circ} \mathrm{C}$ with the following primary antibodies (all from Affinity Biosciences): SIRT1 (1:2,000; cat. no. DF6033), GRP78 (1:800; cat. no. AF5366), eIF2 $\alpha$ (1:1,000; cat. no. AF6087; Affinity Biosciences), phosphorylated (p)-eIF2 $\alpha$ (Ser52) (1:1,500; cat. no. AF3087), PERK (1:1,000; cat. no. AF5304), p-PERK (Thr982) (1:1,500; cat. no. DF7576), CHOP (1:1,500; cat. no. DF6025), caspase-12 (1:1,500; cat. no. AF5199), pro-caspase-3 (1:1,500; cat. no. AF6311), pro-caspase-9 (1:1,500; cat. no. AF6348) or anti- $\beta$-actin (1:5,000; cat. no. AF7018). A goat anti-rabbit secondary antibody (1:5,000; cat. no. IH-0011; Beijing Dingguo Changsheng Biotechnology Co., Ltd.) was used to observe primary antibody binding, and then bands were visualized using an ECL western blotting substrate (cat. no. KF001; Affinity Biosciences). Densitometric analysis of the bands was conducted using ImageJ software (version 1.5.1k; National Institutes of Health).

Statistical analysis. All data are presented as the mean \pm SD and were analyzed using SPSS 22.0 (IBM Corp.). Data were analyzed using one-way ANOVA followed by Tukey's Honest Significant Difference test. $\mathrm{P}<0.05$ was considered to indicate a statistically significant difference.

\section{Results}

IOE improves cardiac function. The cardiac function of post-surgical hearts was evaluated by echocardiography. As shown in Fig. 1A-E, deterioration of cardiac function was induced in the MI/R group, LVIDd and LVIDs increased to $6.13 \pm 0.99$ and $4.25 \pm 0.46 \mathrm{~mm}$, respectively, whereas LVEF and LVFS decreased to $51.75 \pm 6.39$ and $24.13 \pm 5.00 \%$, respectively, compared with the Sham group $(\mathrm{P}<0.01)$. IOE pretreatment increased the recovery of cardiac function in the impaired hearts, which was demonstrated by increased LVEF and LVFS, as well as decreased LVIDd and LVIDs, compared with the $\mathrm{M} / \mathrm{R}$ group $(\mathrm{P}<0.05$ or $\mathrm{P}<0.01)$.

IOE decreases infarct size and attenuates MI/R Injury. NBT staining was used to observe the effects of IOE on the infarction scale. As shown in Fig. 2A, the infarct size reached up to $36.42 \pm 6.67 \%$ in the MI/R group. IOE $(150,300$ and $600 \mathrm{mg} / \mathrm{kg})$ significantly decreased the infarct size compared with the MI/R group $(\mathrm{P}<0.01)$. Furthermore, the activities of myocardial enzymes (LDH, CK-MB and AST) were significantly increased following MI/R injury compared with the Sham group, which further caused myocardial injury $(\mathrm{P}<0.01$; Fig. 2B-D). IOE attenuated $\mathrm{MI} / \mathrm{R}$ injury through inhibiting the activities of myocardial enzymes in MI/R-IOE groups $(\mathrm{P}<0.01)$.

IOE relieves myocardial tissue injury. Myocardial tissues showed partially disordered and disorderly arrangement of 
A

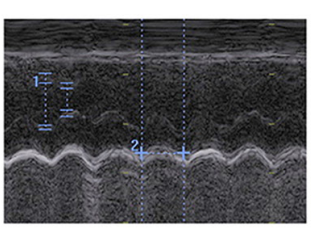

B

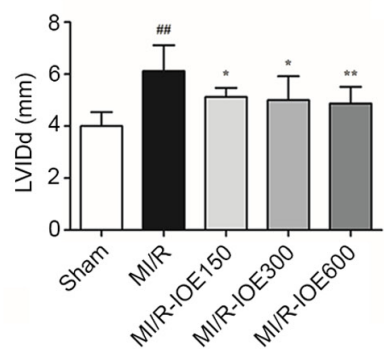

$\mathrm{MI} / \mathrm{R}$

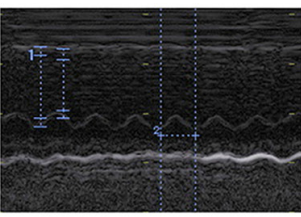

C

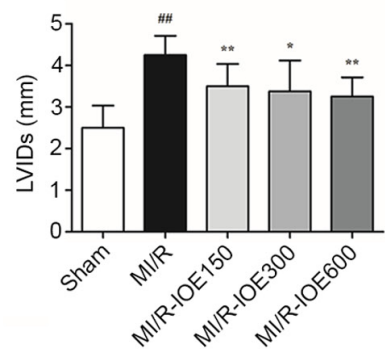

MI/R-IOE150

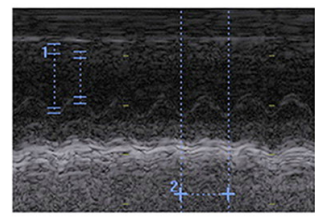

$\mathrm{D}$

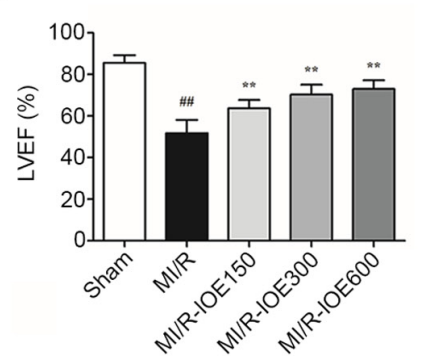

MI/R-IOE600

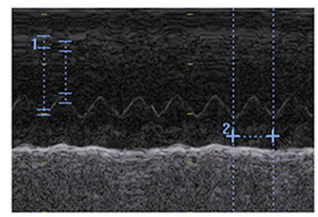

E

Figure 1. IOE improves cardiac function. (A) Echocardiography images of each group. Bar graphs of (B) LVIDd,(C) LVIDs,(D) LVEF and (E) LVFS. Data are presented as the mean \pm standrd deviation. $\mathrm{n}=10 .{ }^{\# \#} \mathrm{P}<0.01$ vs. Sham. ${ }^{*} \mathrm{P}<0.05$ and ${ }^{* *} \mathrm{P}<0.01 \mathrm{vs.} \mathrm{MI/R}$. IOE, Inonotus obliquus extract; LV, left ventricular; LVIDd, LV internal dimensions at diastole; LVIDs, LV internal diameter systole; LVEF, LV ejection fraction; LVFS, LV fractional shortening; MI/R, myocardial ischemia/reperfusion.

A

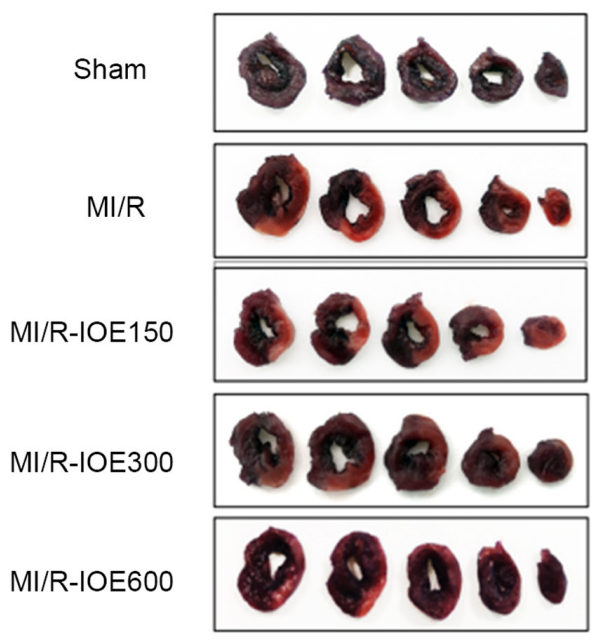

C

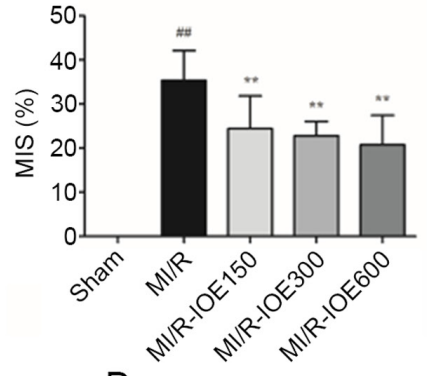

D

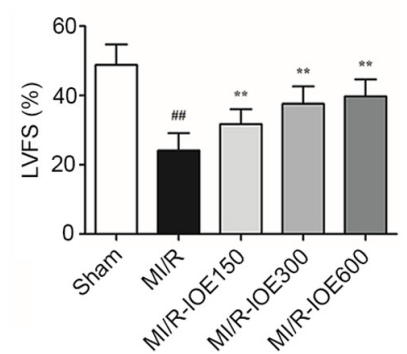

$\mathrm{B}$

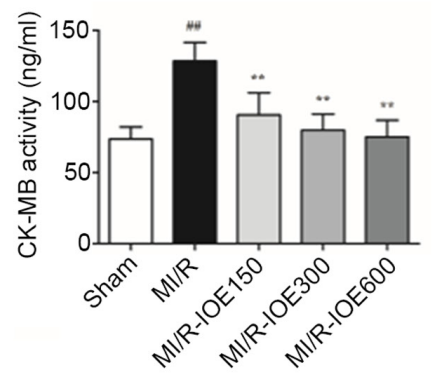

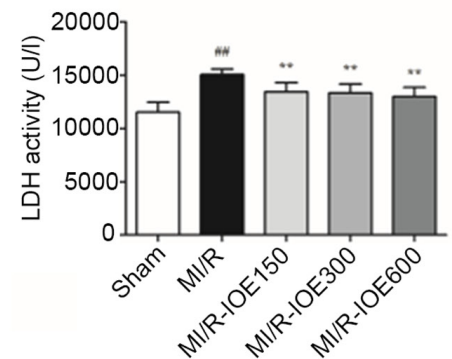

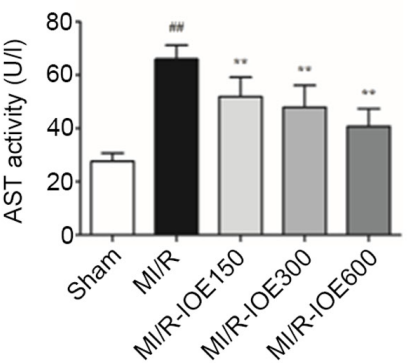

Figure 2. IOE decreases infarct size and attenuates MI/R injury. (A) Representative images of heart tissues. Normal myocardial tissues are dark red and ischemic myocardial tissues are pale. The MIS was calculated as the ischemic myocardial weight/wet weight of left ventricle x100\%. The levels of (B) CK-MB, (C) LDH and (D) AST in serum. Data are presented as the mean \pm standard deviation. $\mathrm{n}=10$. ${ }^{\# \#} \mathrm{P}<0.01$ vs. Sham. ${ }^{* *} \mathrm{P}<0.01 \mathrm{vs}$. MI/R. IOE, Inonotus obliquus extract; MI/R, myocardial ischemia/reperfusion; CK-MB, creatine kinase MB, LDG, lactate dehydrogenase; AST, aminotransferase; MIS, myocardial infarction size.

myocardial fiber stripes, and partial lysis of cardiomyocytes accompanied by interstitial edema in the MI/R group (Fig. 3). Following treatment with IOE, myocardial fibers and cell morphology notably improved to varying degrees.
IOE reduces oxidative stress. MDA content and antioxidant enzymes (SOD, GSH-px and CAT) are key molecules for evaluating the degree of oxidative stress. After MI/R, the activities of these antioxidant enzymes were significantly 
Sham

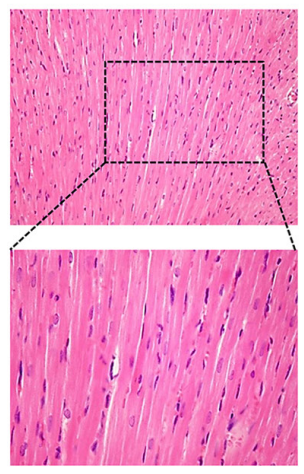

$\mathrm{Ml} / \mathrm{R}$

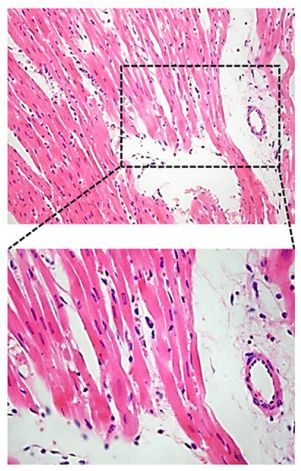

MI/R-IOE150

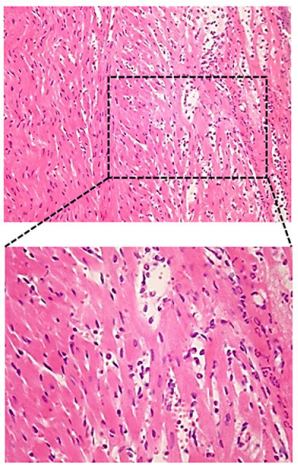

MI/R-IOE300

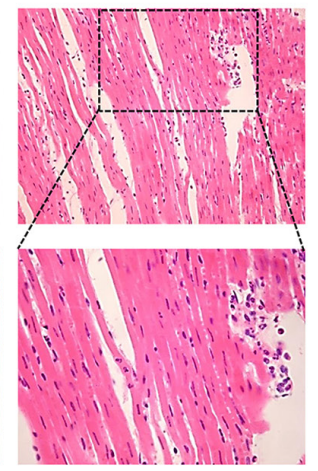

MI/R-IOE600

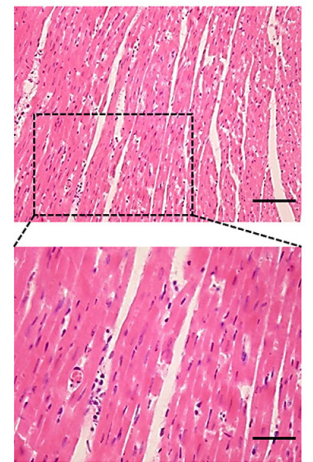

Figure 3. IOE relieves the histopathological changes in myocardial tissue. Representative hematoxylin and eosin staining images of myocardial slices at x200 magnification (upper panel; scale bar, $50 \mu \mathrm{m}$ ) and x400 magnification (lower panel; scale bar, $25 \mu \mathrm{m}$ ). n=4. IOE, Inonotus obliquus extract; MI/R, myocardial ischemia/reperfusion.

A

C
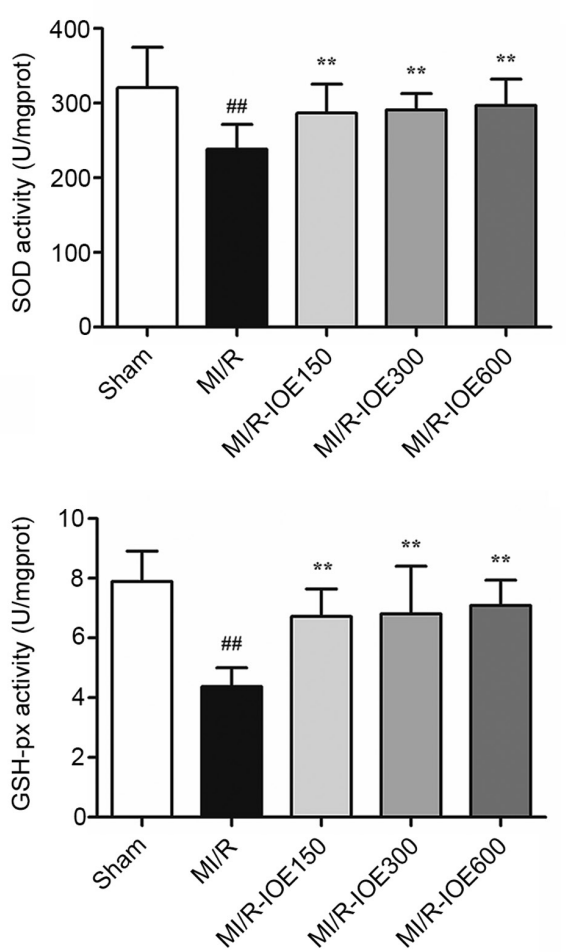

$\mathrm{B}$
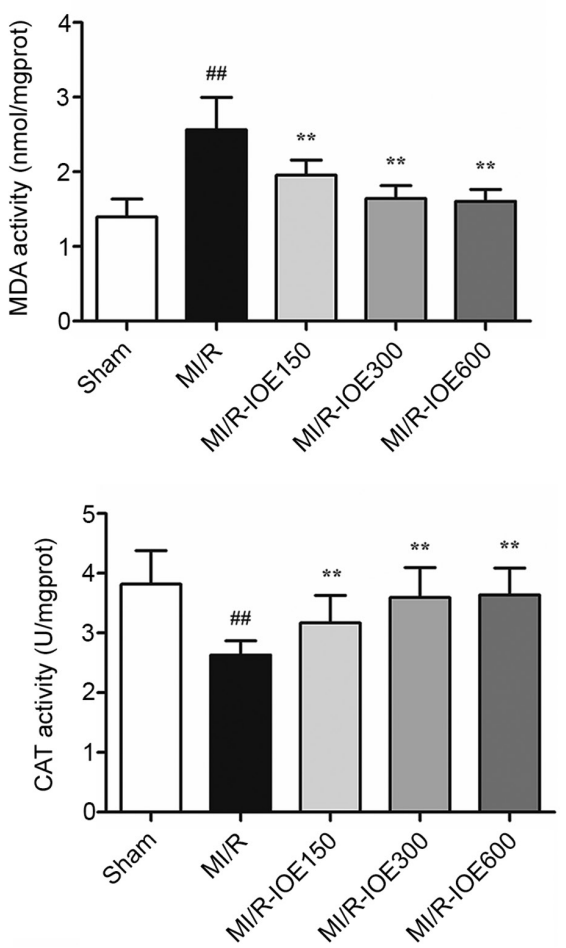

Figure 4. IOE protects cardiomyocytes against oxidative stress. The activity of (A) SOD, the content of (B) MDA, the activity of (C) GSH-px and the activity of (D) CAT. Data are presented as the mean \pm standard deviation. $\mathrm{n}=10 .{ }^{\# \#} \mathrm{P}<0.01$ vs. Sham. ${ }^{* *} \mathrm{P}<0.01$ vs. MI/R. IOE, Inonotus obliquus extract; SOD, superoxide dismutase; GSH-px, glutathione peroxidase; CAT, catalase; MDA, malondialdehyde; MI/R, myocardial ischemia/reperfusion.

reduced (Fig. 4A, C and D). Meanwhile, the content of MDA was significantly higher compared with the Sham group $(\mathrm{P}<0.01$; Fig. 4B). IOE pretreatment $(150,300$ and $600 \mathrm{mg} / \mathrm{kg})$ increased the activities of antioxidant enzymes and decreased MDA content compared with the MI/R group $(\mathrm{P}<0.01)$. These values indicated that IOE pretreatment protected cardiomyocytes against oxidative stress.

IOE decreases cardiomyocyte apoptosis. The present study aimed to detect the role of IOE in cell death by TUNEL staining. MI/R injury induced a certain degree of cardiomyocyte apoptosis by increasing the percentage of apoptotic cells in the MI/R group compared with the Sham group $(\mathrm{P}<0.01$;
Fig. 5A and B). As expected, pretreatment with IOE (150, 300 and $600 \mathrm{mg} / \mathrm{kg}$ ) significantly decreased the percentage of apoptotic cells $(\mathrm{P}<0.05$ or $\mathrm{P}<0.01)$. Therefore, the data suggested that IOE could alleviate cardiomyocyte apoptosis after MI/R injury.

IOE activates SIRTI and downregulates the PERK/eIF $2 \alpha / C H O P$ pathway. Considering the role of SIRT1 in cardioprotection, SIRT1 expression in heart tissue was examined. MI/R injury could suppress SIRT1 expression compared with the Sham group, which was then activated by IOE ( $\mathrm{P}<0.01$; Fig. 6A and B). Furthermore, upregulation of ER chaperone GRP78, p-PERK, p-eIF2 $\alpha$, CHOP and caspase-12 
A

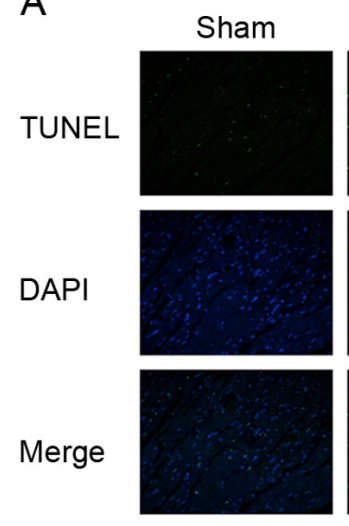

MI/R

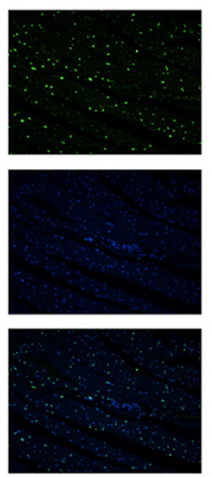

MI/R-IOE150
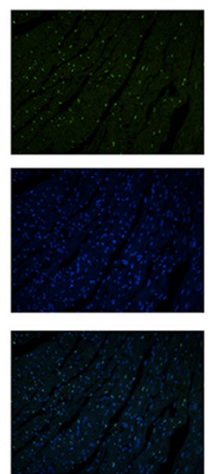

MI/R-IOE300
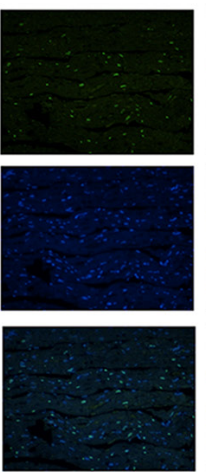

MI/R-IOE600

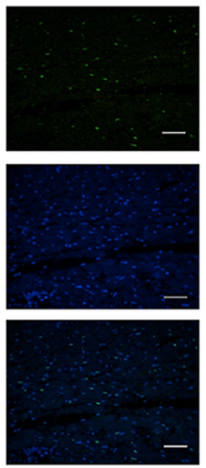

B

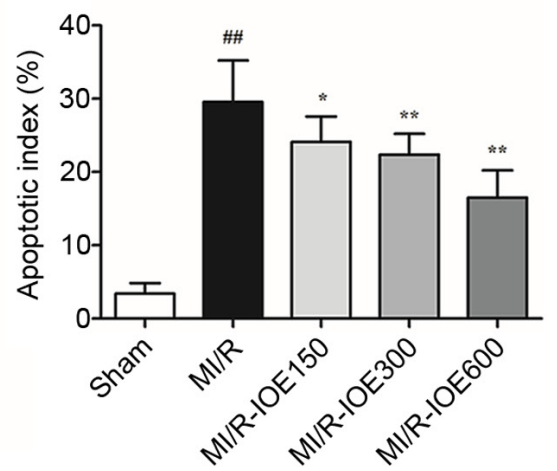

Figure 5. IOE decreases cardiomyocyte apoptosis. (A) Representative images of TUNEL staining (scale bar, $100 \mu \mathrm{m}$ ). (B) Cardiomyocyte apoptosis index. Data are presented as the mean \pm standard deviation. $\mathrm{n}=4 .{ }^{\# \#} \mathrm{P}<0.01$ vs. Sham. ${ }^{*} \mathrm{P}<0.05,{ }^{* *} \mathrm{P}<0.01$ vs. MI/R. IOE, Inonotus obliquus extract; MI/R, myocardial ischemia/reperfusion.

A

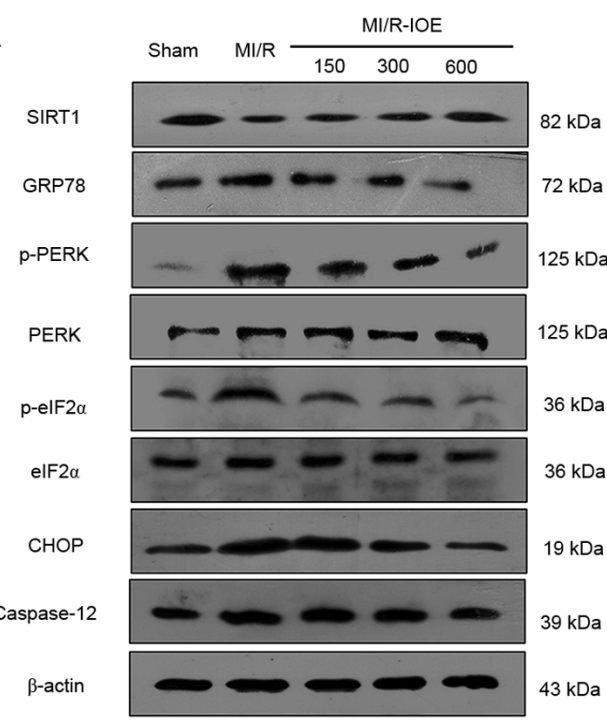
$43 \mathrm{kDa}$
B
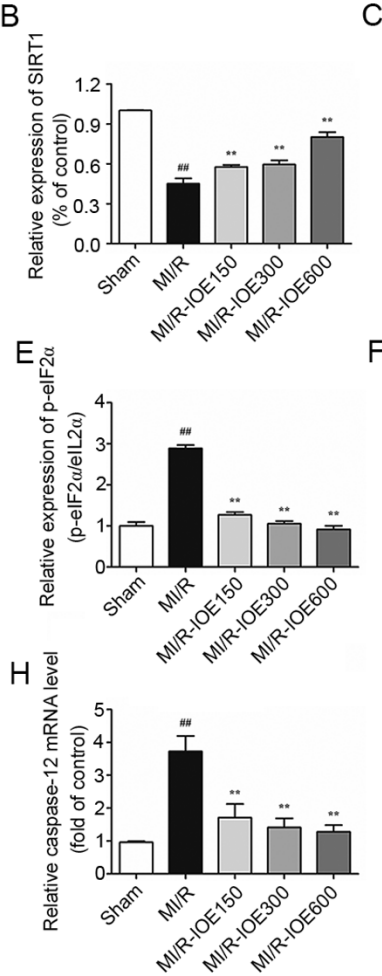

C
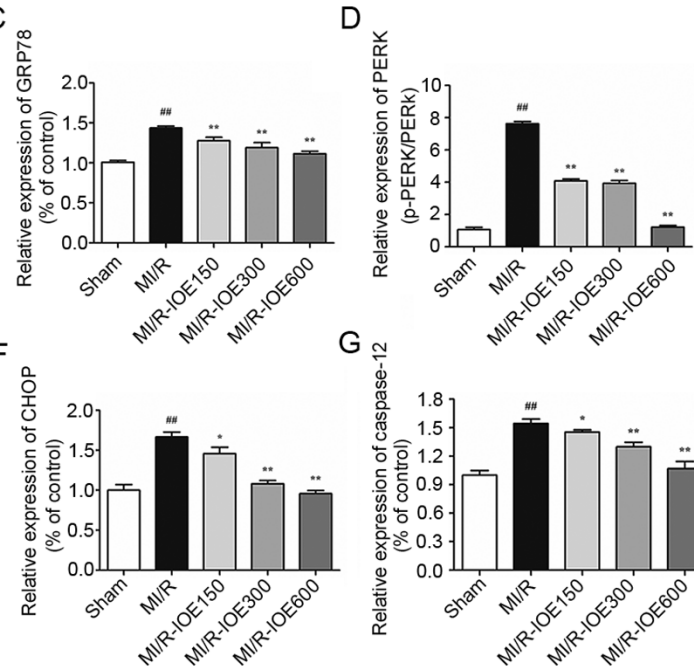

$G_{-}$

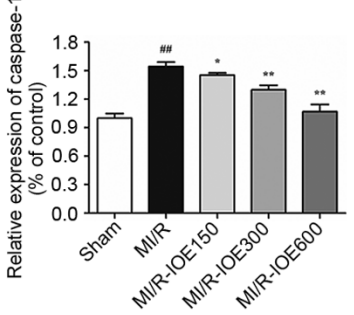

Figure 6. Expression of SIRT1, GRP78, p-PERK, p-eIF2 $\alpha$, CHOP, caspase-12 and the mRNA levels of caspase-12 in the myocardium. (A) Representative blots of SIRT1, GRP78, p-PERK, p-eIF2 $\alpha$, CHOP and caspase-12. Semiquantitative analysis of (B) SIRT1, (C) GRP78, (D) p-PERK, (E) p-eIF2 $\alpha$, (F) CHOP and $(\mathrm{G})$ caspase-12. $(\mathrm{H})$ The mRNA levels of caspase-12 in the myocardium. Data are presented as the mean \pm standard deviation. $\mathrm{n}=3$. ${ }^{\# \prime} \mathrm{P}<0.01 \mathrm{vs}$. Sham. ${ }^{*} \mathrm{P}<0.05$ and ${ }^{* *} \mathrm{P}<0.01$ vs. MI/R. IOE, Inonotus obliquus extract; SIRT1, NAD-dependent protein deacetylase sirtuin-1; GRP78, glucose-regulated protein 78; PERK, protein kinase R-like endoplasmic reticulum kinase; eIF2 $\alpha$, eukaryotic translation initiation factor 2 subunit $\alpha$; CHOP, C/EBP homologous protein; p, phosphorylated; MI/R, myocardial ischemia/reperfusion.

was observed after $\mathrm{MI} / \mathrm{R}$ injury in the heart $(\mathrm{P}<0.01$; Fig. 6A and C-G). Moreover, the changes in mRNA expression of caspase-12 were investigated. As shown in Fig. $6 \mathrm{H}$, the mRNA levels of caspase-12 in the ischemic myocardium were also notably increased in the MI/R group compared with the Sham group $(\mathrm{P}<0.01)$. As expected, IOE inhibited ER stress by downregulating the expression of ER stress-related markers (GRP78, p-PERK, p-eIF2 $\alpha$, CHOP and caspase-12) compared with the $\mathrm{MI} / \mathrm{R}$ group $(\mathrm{P}<0.05$ or $\mathrm{P}<0.01)$. IOE decreased the mRNA levels of caspase-12 in the MI/R-IOE groups $(\mathrm{P}<0.01)$.
Collectively, the aforementioned values suggested that IOE attenuated MI/R injury by suppressing ER stress, which may be associated with SIRT1 activation.

IOE suppresses ER stress-induced apoptosis. To evaluate the condition of cardiomyocyte apoptosis, the expression of pro-caspase- 9 and pro-caspase- 3 in ischemic hearts were measured. MI/R injury significantly decreased the expression of pro-caspase- 9 and pro-caspase- 3 compared with the Sham group $(\mathrm{P}<0.01$; Fig. 7A-C). By contrast, IOE increased 
A

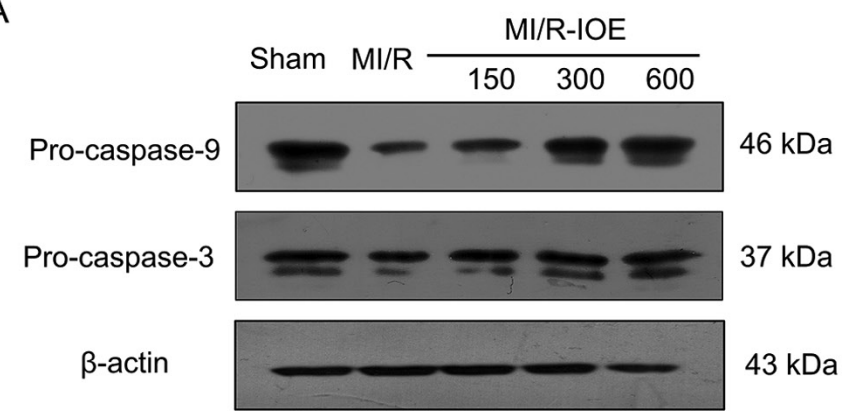

B

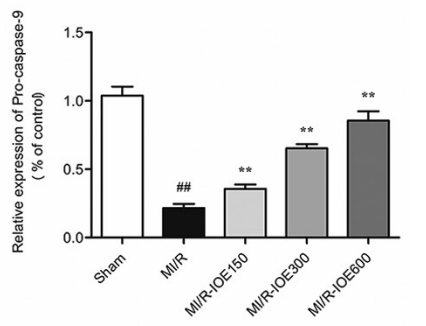

C

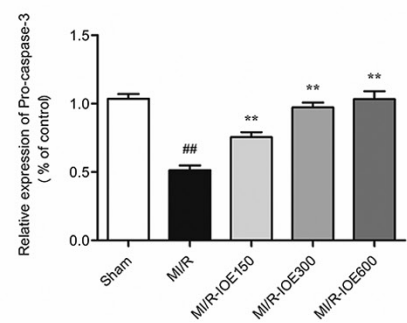

Figure 7. Expression of pro-caspase-9 and pro-caspase-3 in the myocardium. (A) Representative blots of pro-caspase-9 and pro-caspase-3. Semiquantitative analysis of (B) pro-caspase-9 and (C) pro-caspase-3. Data are presented as the mean \pm standard deviation. $\mathrm{n}=3$. ${ }^{\#} \mathrm{P}<0.01 \mathrm{vs}$. Sham. ${ }^{* *} \mathrm{P}<0.01 \mathrm{vs}$. $\mathrm{MI} / \mathrm{R}$. $\mathrm{MI} / \mathrm{R}$, myocardial ischemia/reperfusion; IOE, Inonotus obliquus extract.

the expression of pro-caspase-9 and pro-caspase- 3 in ischemic heart tissue compared with the MI/R group $(\mathrm{P}<0.05$ or $\mathrm{P}<0.01)$. The data indicated that IOE protected the heart from $\mathrm{MI} / \mathrm{R}$ injury via suppressing ER stress-induced cardiomyocyte apoptosis.

\section{Discussion}

IO possesses various pharmacological and biological properties, including antitumor, antimicrobial and antioxidant activities (19). It was previously reported that IO inhibited lipid peroxidation in hyperlipidemic rats (20). In the present study, IOE exerted cardioprotective effects by attenuating oxidative damage and suppressing ER stress-induced apoptosis.

Redox signaling is crucial in the regulation of cell function, including defense against invading microorganisms and gene expression for physiological activity. MI/R induces excessive generation of redox signaling of ROS to break redox homeostasis, which results in oxidative stress (21). The severity of reversible and irreversible cellular injury within cardiomyocytes is proportional to the level of ROS activation (22). ROS can induce lipid peroxidation, protein oxidation and nitration, mitochondrial permeability transition and DNA damage, causing cardiomyocyte death following MI/R (23). To keep the redox state in balance, redundant ROS is typically eliminated by several enzymes, such as SOD, GSH-px and CAT (24). Several therapeutic strategies can protect cardiomyocytes by inhibiting oxidative stress, for example, pre-conditioning, pro-conditioning and antioxidants (23). Our previous study revealed that ginsenoside $\mathrm{Rg} 2$ protected cardiomyocytes from MI/R injury by inhibiting oxidative stress and inflammation (25). In the present study, MI/R injury induced downregulation of GSH-px, SOD and CAT activities and upregulation of MDA content, which were counteracted by IOE. These results indicated that IOE pretreatment protected cardiomyocytes against oxidative stress.

Ischemia and reperfusion activate a variety of cell death programs, categorized as apoptosis, necrosis or autophagy-associated cell death (26). ER stress plays a vital role in the pathogenesis of cardiac diseases and may be a therapeutic target to protect cardiomyocytes. In the MI/R process, ER stress occurs due to diverse stimuli, and then triggers the unfolded protein response (UPR). Overproduction of ROS disrupts protein folding and results in amplified UPR signaling (27). Previous evidence has suggested that UPR is initially activated by three endoplasmic reticulum stress sensors, serine/threonine-protein kinase/endoribonuclease IRE1, PERK and ATF6 (28). GRP78, an immunoglobulin binding protein, physically binds to these proteins and inhibits their activities under a steady state (29). PERK, also known as eukaryotic initiation factor $2 \alpha$ protein kinase 3, interacts with and phosphorylates eIF2 $\alpha$ (30). Under ER stress, PERK dissociates from GRP78 and induces the phosphorylation of eIF $2 \alpha$. The PERK/eIF $2 \alpha$ signaling pathway can limit protein translation and induce apoptosis by inducing CHOP expression (31). Caspase-12 localizes in the ER and is a critical mediator in ER stress-induced apoptosis by activating other caspases (32). Caspase-12 specifically clears pro-caspase-9 for activation and caspase-9 is active. Subsequently, caspase-9 ultimately activates caspase-3, the major effector caspase for apoptosis (33). In the present study, MI/R injury induced ER stress in rat cardiomyocytes. It was found that IOE pretreatment significantly decreased the expression of GRP78, p-PERK, p-eIF $2 \alpha$, CHOP and caspase-12 and the mRNA levels of Caspase-12. The present study also found that ER stress-induced apoptosis by MI/R injury was alleviated by IOE. Above all, the cardioprotective effects of IOE are based on the suppression of ER stress-induced cardiomyocyte apoptosis.

SIRT1 is a highly conserved histone deacetylase, which can deacetylate various transcription factors (34). It has been demonstrated that SIRT1 activation has protective effects in myocardial ischemia. SIRT1 and ER stress are involved in various pathologies, including diabetes, obesity, cancer and cardiovascular diseases $(4,35,36)$. Resveratrol, a SIRT1 activator, inhibits ER stress via SIRT1 activation in vitro (37). Previous studies have indicated that SIRT1 may regulate eIF $2 \alpha$ phosphorylation via regulating its acetylation process, thereby affecting its activity $(38,39)$. SIRT1 inhibition has been demonstrated to cause the hyperactivation of the PERK/eIF2 $\alpha$ pathway, which induces cardiomyocyte apoptosis by sustaining CHOP expression (12). The present study revealed that IOE notably increased SIRT1 expression in the ischemic myocardium. IOE also suppressed ER stress-induced apoptosis by mediating the PERK/eIF $2 \alpha / \mathrm{CHOP}$ signaling pathway. The present results demonstrated that IOE-induced SIRT1 activation may suppress ER stress-induced cardiomyocyte apoptosis, thereby reducing the infarct size in vivo. However, there is a complex interaction between SIRT1 and ER stress. SIRT1 activation has been reported to inhibit ER stress and 
apoptosis of cardiomyocytes by promoting autophagic clearance and decreasing the expression of the protein disulfide isomerase and S-nitrosylation $(40,41)$. The role of IOE in these mechanisms need to be further studied.

In conclusion, the present findings showed that IOE pretreatment protected cardiomyocytes from MI/R injury by attenuating oxidative damage and suppressing ER stress-induced apoptosis. IOE may suppress ER stress-induced cardiomyocyte apoptosis via the PERK/eIF2 $\alpha /$ CHOP pathway by the activation of SIRT1. Therefore, IO may be a potential therapeutic candidate in cardiovascular diseases.

\section{Acknowledgements}

Not applicable.

\section{Funding}

This project was supported by the Jilin Scientific and Technological Development Program (grant no. 20190701056GH).

\section{Availability of data and materials}

The datasets used and/or analyzed during the current study are available from the corresponding author on reasonable request.

\section{Authors' contributions}

YX and WF conceived the study. YW, HC, YZ, PY, YL, DW, YX and WF performed the experiments. HC, YZ, PY, YL and DW analyzed the data. YW, YX and WF wrote the manuscript. YX and WF improved the manuscript. All authors read and approved the final manuscript.

\section{Ethics approval and consent to participate}

The animal experiments of this study were approved by the Ethical Committee for Experimental Animals, School of Pharmaceutical Sciences Jilin University (approval no. 20190049; Changchun, China).

\section{Patient consent for publication}

Not applicable.

\section{Competing interests}

The authors declare that they have no competing interests.

\section{References}

1. Lu D and Thum T: RNA-based diagnostic and therapeutic strategies for cardiovascular disease. Nat Rev Cardiol 16 : 661-674, 2019.

2. Robin E, Guzy RD, Loor G, Iwase H, Waypa GB, Marks JD, Hoek TL and Schumacker PT: Oxidant stress during simulated ischemia primes cardiomyocytes for cell death during reperfusion. J Biol Chem 282: 19133-19143, 2007.

3. Wang J, Hu X and Jiang H: ER stress-induced apoptosis: A novel therapeutic target in myocardial ischemia and reperfusion injury. Int J Cardiol 214: 233-234, 2016.
4. Oakes SA and Papa FR: The role of endoplasmic reticulum stress in human pathology. Annu Rev Pathol 10: 173-194, 2015.

5. Cao SS and Kaufman RJ: Endoplasmic reticulum stress and oxidative stress in cell fate decision and human disease. Antioxid Redox Signal 21: 396-413, 2014.

6. Lee AS: GRP78 induction in cancer: Therapeutic and prognostic implications. Cancer Res 67: 3496-3499, 2007.

7. Harding HP, Zhang Y and Ron D: Protein translation and folding are coupled by an endoplasmic-reticulum-resident kinase. Nature 397: 271-274, 1999.

8. Harding HP, Novoa I, Zhang Y, Zeng H, Wek R, Schapira M and Ron D: Regulated translation initiation controls stress-induced gene expression in mammalian cells. Mol Cell 6: 1099-1108, 2000.

9. Li X, Wang Y, Wang H, Huang C, Huang Y and Li J: Endoplasmic reticulum stress is the crossroads of autophagy, inflammation, and apoptosis signaling pathways and participates in liver fibrosis. Inflamm Res 64: 1-7, 2015.

10. Singh V and Ubaid S: Role of Silent Information Regulator 1 (SIRT1) in Regulating Oxidative Stress and Inflammation. Inflammation 43: 1589-1598, 2020.

11. Luo G, Jian Z, Zhu Y, Zhu Y, Chen B, Ma R, Tang F and Xiao Y: Sirt 1 promotes autophagy and inhibits apoptosis to protect cardiomyocytes from hypoxic stress. Int J Mol Med 43: 2033-2043, 2019.

12. Prola A, Pires Da Silva J, Guilbert A, Lecru L, Piquereau J, Ribeiro M, Mateo P, Gressette M, Fortin D, Boursier C, et al: SIRT1 protects the heart from ER stress-induced cell death through eIF2 $\alpha$ deacetylation. Cell Death Differ 24: 343-356, 2017.

13. Wasser SP: Medicinal Mushroom Science: History, Current Status, Future Trends, and Unsolved Problems. Int J Med Mushrooms 12: 1-16, 2010.

14. Lee I-K and Yun B-S: Styrylpyrone-class compounds from medicinal fungi Phellinus and Inonotus spp., and their medicinal importance. J Antibiot (Tokyo) 64: 349-359, 2011.

15. Javed S, Mitchell K, Sidsworth D, Sellers SL, Reutens-Hernandez J, Massicotte HB, Egger KN, Lee $\mathrm{CH}$ and Payne GW: Inonotus obliquus attenuates histamine-induced microvascular inflammation. PLoS One 14: e0220776, 2019.

16. Fan L, Ding S, Ai L and Deng K: Antitumor and immunomodulatory activity of water-soluble polysaccharide from Inonotus obliquus. Carbohydr Polym 90: 870-874, 2012.

17. National Research Council (US) Committee for the Update of the Guide for the Care and Use of Laboratory Animals: Guide for the Care and Use of Laboratory Animals. 8th edition. National Academies Press, Washington, DC, 2011.

18. Livak KJ and Schmittgen TD: Analysis of relative gene expression data using real-time quantitative PCR and the $2(\mathrm{~T})$ (-Delta Delta C) method. Methods 25: 402-408, 2001.

19. Balandaykin ME and Zmitrovich IV: Review on Chaga Medicinal Mushroom, Inonotus obliquus (Higher Basidiomycetes): Realm of Medicinal Applications and Approaches on Estimating its Resource Potential. Int J Med Mushrooms 17: 95-104, 2015.

20. Liang L, Zhang Z, Sun W and Wang Y: Effect of the Inonotus obliquus polysaccharides on blood lipid metabolism and oxidative stress of rats fed high-fat diet in vivo. In: Proceedings of the 2009 2nd International Conference on Biomedical Engineering and Informatics. IEEE, New York, NY, pp1114-1117, 2009.

21. Kalogeris T, Baines CP, Krenz M and Korthuis RJ: Ischemia/Reperfusion. Compr Physiol 7: 113-170, 2016.

22. Horwitz LD, Wallner JS, Decker DE and Buxser SE: Efficacy of lipid soluble, membrane-protective agents against hydrogen peroxide cytotoxicity in cardiac myocytes. Free Radic Biol Med 21: 743-753, 1996.

23. Raedschelders K, Ansley DM and Chen DD: The cellular and molecular origin of reactive oxygen species generation during myocardial ischemia and reperfusion. Pharmacol Ther 133: 230-255, 2012.

24. He L, He T, Farrar S, Ji L, Liu T and Ma X: Antioxidants Maintain Cellular Redox Homeostasis by Elimination of Reactive Oxygen Species. Cell Physiol Biochem 44: 532-553, 2017.

25. Fu WW, Xu HL, Yu XF, Lyu C, Tian Y, Guo M, Sun J and Sui D: 20(S)-Ginsenoside Rg2 attenuates myocardial ischemia/reperfusion injury by reducing oxidative stress and inflammation: Role of SIRT1. Rsc Adv 8: 23947-23962, 2018.

26. Hotchkiss RS, Strasser A, McDunn JE and Swanson PE: Cell death. N Engl J Med 361: 1570-1583, 2009.

27. Malhotra JD, Miao H, Zhang K, Wolfson A, Pennathur S, Pipe SW and Kaufman RJ: Antioxidants reduce endoplasmic reticulum stress and improve protein secretion. Proc Natl Acad Sci USA 105: 18525-18530, 2008. 
28. Hetz C: The unfolded protein response: Controlling cell fate decisions under ER stress and beyond. Nat Rev Mol Cell Biol 13: 89-102, 2012.

29. Louessard M, Bardou I, Lemarchand E, Thiebaut AM, Parcq J, Leprince J, Terrisse A, Carraro V, Fafournoux P, Bruhat A, et al: Activation of cell surface GRP78 decreases endoplasmic reticulum stress and neuronal death. Cell Death Differ 24: 1518-1529, 2017.

30. Taniuchi S, Miyake M, Tsugawa K, Oyadomari M and Oyadomari S: Integrated stress response of vertebrates is regulated by four eIF2 $\alpha$ kinases. Sci Rep 6: 32886, 2016.

31. Tabas I and Ron D: Integrating the mechanisms of apoptosis induced by endoplasmic reticulum stress. Nat Cell Biol 13: 184-190, 2011

32. Nakagawa T, Zhu H, Morishima N, Li E, Xu J, Yankner BA and Yuan J: Caspase-12 mediates endoplasmic-reticulum-specific apoptosis and cytotoxicity by amyloid-beta. Nature 403: 98-103, 2000.

33. Fan TJ, Han LH, Cong RS and Liang J: Caspase family proteases and apoptosis. Acta Biochim Biophys Sin (Shanghai) 37: 719-727, 2005.

34. Yang Y, Duan W, Li Y, Jin Z, Yan J, Yu S and Yi D: Novel role of silent information regulator 1 in myocardial ischemia. Circulation 128: 2232-2240, 2013.

35. Hetz C, Chevet E and Harding HP: Targeting the unfolded protein response in disease. Nat Rev Drug Discov 12: 703-719, 2013

36. Winnik S, Auwerx J, Sinclair DA and Matter CM: Protective effects of sirtuins in cardiovascular diseases: From bench to bedside. Eur Heart J 36: 3404-3412, 2015.
37. Liu LQ, Fan ZQ, Tang YF and Ke ZJ: The resveratrol attenuates ethanol-induced hepatocyte apoptosis via inhibiting ER-related caspase-12 activation and PDE activity in vitro. Alcohol Clin Exp Res 38: 683-693, 2014.

38. Hubbard BP, Gomes AP, Dai H, Li J, Case AW, Considine T, Riera TV, Lee JE, E SY, Lamming DW, et al: Evidence for a common mechanism of SIRT1 regulation by allosteric activators. Science 339: 1216-1219, 2013

39. Ron D and Walter P: Signal integration in the endoplasmic reticulum unfolded protein response. Nat Rev Mol Cell Biol 8: 519-529, 2007.

40. Pires Da Silva J, Monceaux K, Guilbert A, Gressette M, Piquereau J, Novotova M, Ventura-Clapier R, Garnier A and Lemaire C: SIRT1 Protects the Heart from ER Stress-Induced Injury by Promoting eEF2K/eEF2-Dependent Autophagy. Cells 9: 426, 2020.

41. Hsu YJ, Hsu SC, Hsu CP, Chen YH, Chang YL, Sadoshima J, Huang SM, Tsai CS and Lin CY: Sirtuin 1 protects the aging heart from contractile dysfunction mediated through the inhibition of endoplasmic reticulum stress-mediated apoptosis in cardiac-specific Sirtuin 1 knockout mouse model. Int J Cardiol 228: 543-552, 2017

This work is licensed under a Creative Commons Attribution-NonCommercial-NoDerivatives 4.0 International (CC BY-NC-ND 4.0) License. 